\title{
Micro-Opto-Mechanical sensors for tactile width measurements of surface opening cracks in concrete
}

\author{
Diego Marini, Luca Belsito, Fulvio Mancarella, Filippo Bonafè, Alberto Roncaglia \\ Institute for Microelectronics and Microsystems (IMM) \\ National Research Council of Italy (CNR) \\ Bologna, Italy \\ marini@bo.imm.cnr.it
}

\begin{abstract}
The fabrication and laboratory testing of MicroOpto-Mechanical sensors for the measurements of width of surface opening cracks in concrete structures are presented. The sensors are designed to be operated on a fan-out connector containing 12 multimode optical fibers, using a microstructure that can be mounted on the connector with a self aligned procedure. Thanks to the optical alignment automatically provided by such mounting method, each fiber in the connector can be used to interrogate an individual pressure sensor within an array composed by 12 elements in total. Using a soft polymer layer between the microsensor and the crack opening, the width of the crack can be derived by means of a tactile measurement performed by interrogating the 12 elements of the array alternatively, with a space resolution of $0.25 \mathrm{~mm}$. The device is designed to be operated on board of a Remotely Piloted Aerial Vehicle equipped with a vision system that will be able to locate surface cracks of the external surface of reinforced concrete bridges and perform contact measurements on cracks using a robotic arm operated from the vehicle.
\end{abstract}

Keywords-Micro-Opto-Mechanical Systems; surface cracks; tactile measurements

\section{INTRODUCTION}

In structural assessment of infrastructures, the measurement of width and depth of surface opening cracks is a routinely employed method, normally performed manually, since from these data structural engineers can evaluate the severity of damage on the structure and decide possible consolidation interventions. Crack depth is generally measured with ultrasonic methods that require to access the surface opening crack from a close distance $[1,2]$ whereas width is measured visually with the aid of special rulers possibly equipped with optical magnifying systems. In both cases, the intervention of a human operator on the location of the surface opening crack is needed to take these measurements.

Since buildings and infrastructures on which such measurements are needed are often difficult to access, the possibility to execute these measurements using an autonomous robot represents a vey attractive perspective, since this may allow to reduce inspection costs and avoid possible dangers to human operators.

In a previous paper [3], we reported about the development of an ultrasonic sensor system that can be used automatically on board of a terrestrial robot to measure the depth of surface opening crack on the lining of road tunnels with ultrasonic methods. In this paper, a tactile sensor for the measurement of surface crack width based on a Micro-Opto-Mechanical System (MOMS) technology with a completely optical readout is presented. The device is designed to be operated by a Remotely Piloted Aerial Vehicle (RPAV) equipped with a robotic arm that will be used for bridge inspection.

\section{SENSOR DESIGN AND FABRICATION}

\section{A. Sensor design and principle of operation}

The design of the sensor is schematically represented in Fig. 1. It is composed by an array of thin membrane pressure sensor manufactured on a silicon micromechanical frame that can be used to mount the device on a fan-out multiple optical connector composed by 12 optical fibers.

As can be seen from the figure, the device contains a MOMS pressure sensor array operating based on the reflectance modulation of the Fabry-Perot optical cavity created by the space between the termination of the fibers in the fan-out connector and the pressure sensing membrane of the MOMS sensor. Each cavity in the array can be separately interrogated by a laser beam driven through one of the fibers of the fan-out connector in order to measure the deflection of the membranes induced by the applied pressure.
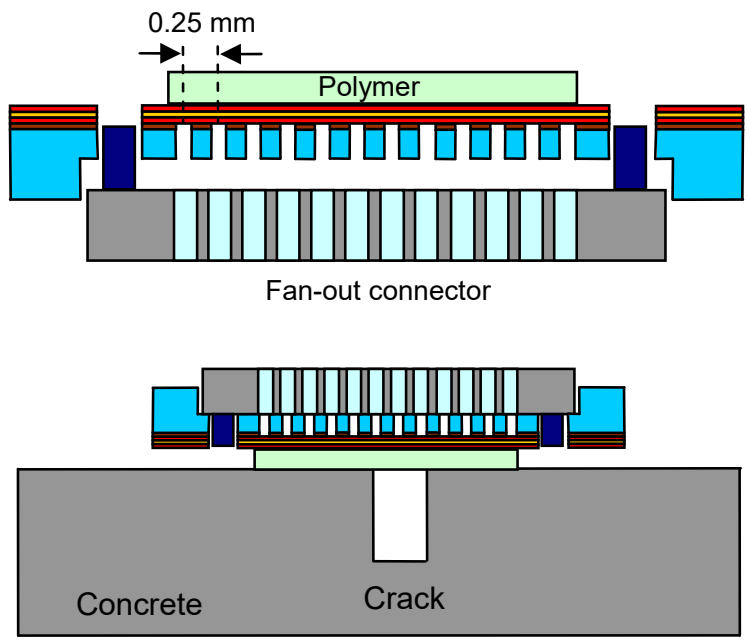

Fig. 1. Cross section (top) and operating principle (bottom) of the opto-mechanical sensor for surface crack width measurements. 
As reported in Fig. 1 bottom, the measurement is performed by putting the fan-out optical sensors in contact with the crack. Because of the array structure of the sensor, by placing the device across the crack on the surface of concrete, some of the sensing pixels will be located over the crack opening, and consequently not in contact with concrete, and some other outside the opening, therefore in contact with concrete.

By applying some pressure over the tool, only the individual pressure sensors in contact with concrete will sense a significant pressure, whereas those over the crack opening will have essentially no signal. In both cases, by analyzing the intensity of the sensing signals over the array of pixels, an estimation of the crack depth will be possible, with a resolution depending on the pitch of the pixel array, in turn dependent on the one of the fan-out connector $(0.25 \mathrm{~mm})$.

In order to evaluate the expected opto-mechanical sensitivity of the sensor, some finite-element simulations were carried out, in which the expected deflection of a thin membrane (circular or rectangular) composed by a multiple stack of polycrystalline silicon and silicon nitride was calculated. With the aid of the simulations, the design parameters reported in Tab. 1 were chosen in the design.

Tab 1. Design parameters of the optomechanical pressure sensors.

\begin{tabular}{|c|c|}
\hline Design parameter & Values \\
\hline \hline Mounting pin hole diameter & $710 \mu \mathrm{m}$ \\
\hline Optical fiber hole diameter (circular) & $120,140,160 \mu \mathrm{m}$ \\
\hline Optical fiber hole width (rectangular) & $100,120,140,160 \mu \mathrm{m}$ \\
\hline Diaphragm thickness (d) & $100 \mu \mathrm{m}$
\end{tabular}

\section{B. Fabrication}

The crack width measurement module prototype was manufactured according to the fabrication process illustrated in Fig. 2.

1. Si substrate

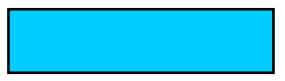

5. $\mathrm{SiO}_{2} /$ poly/ $/ \mathrm{Si}_{3} \mathrm{~N}_{4}$ etching

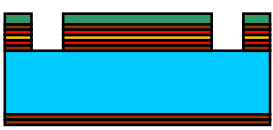

9. Si etching

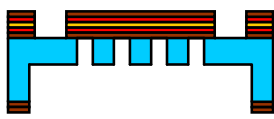

2. $\mathrm{SiO}_{2} /$ poly $/ \mathrm{Si}_{3} \mathrm{~N}_{4}$ deposition

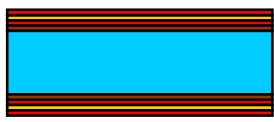

6. $\mathrm{SiO}_{2}$ etching

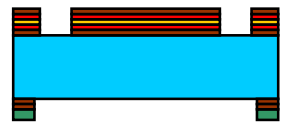

10. $\mathrm{SiO}_{2}$ deposition

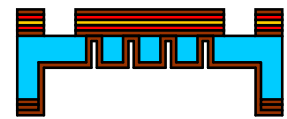

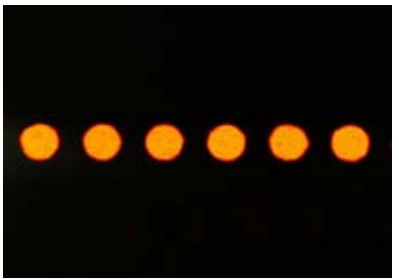
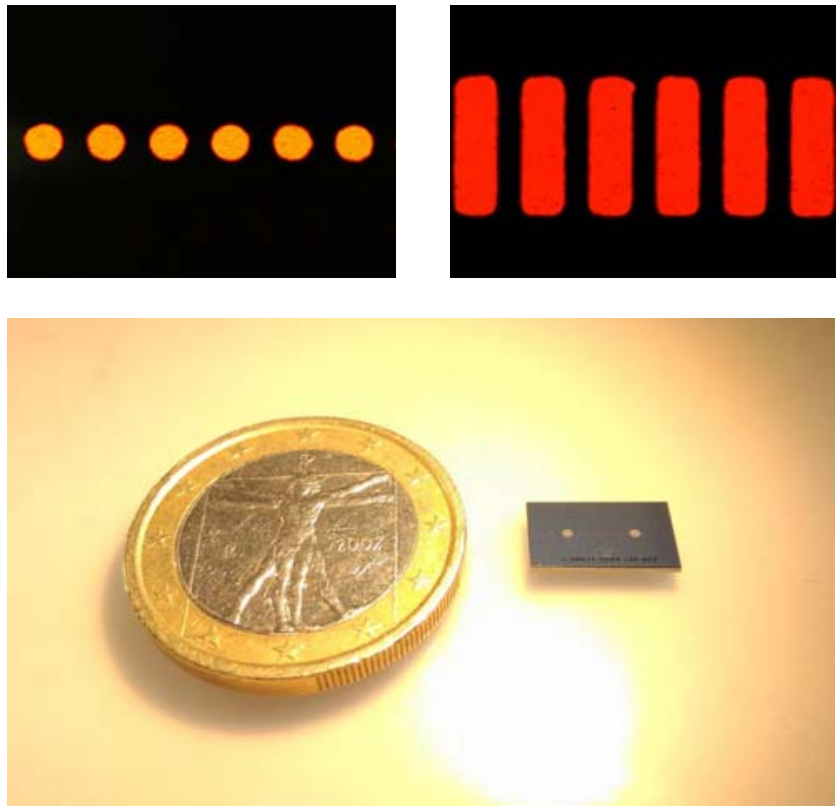

Fig. 3. Membrane arrays observed at the optical microscope (top) and sensor prototype compared with a one euro coin (bottom).

Starting from a $500 \mu \mathrm{m}$ thick silicon substrate, a polysilicon/silicon nitride triple stack $(1 \mu \mathrm{m}$ thick bottom polysilicon, $300 \mathrm{~nm}$ thick $\mathrm{Si}_{3} \mathrm{~N}_{4}, 1 \mu \mathrm{m}$ thick top polysilicon) is deposited over a $2 \mu \mathrm{m}$ thick $\mathrm{SiO}_{2}$ layer, all deposited by LowPressure Chemical Vapour Deposition (LPCVD) and subsequently removed from the backside of the wafer (steps 2 , 3). Afterwards, the substrate is micromachined from the backside by Deep Reactive Ion Etching (DRIE) in order to create the pressure sensing membranes and the cavity used to accommodate the devices on the fan-out connector (steps 4-9). An additional front-side DRIE micromachining is finally executed to create the pass-through mounting holes on the MOMS (steps 10-12). Some images of the fabricated devices are reported in Fig. 3.

3. Poly/ $/ \mathrm{Si}_{3} \mathrm{~N}_{4}$ etching

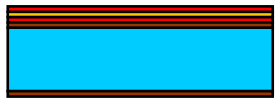

7. Si etching

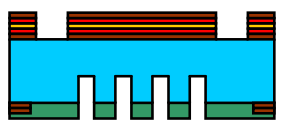

11. Si etching

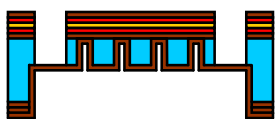

4. $\mathrm{SiO}_{2}$ deposition

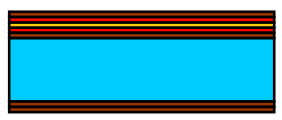

8. $\mathrm{SiO}_{2} / \mathrm{poly} / \mathrm{Si}_{3} \mathrm{~N}_{4}$ etching

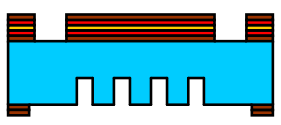

12. $\mathrm{SiO}_{2}$ etching

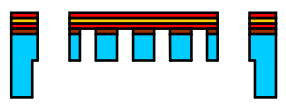

Fig. 2. Process flow for the manufacturing of the sensors. 


\section{TESTING}

The prototypes obtained in this way were tested once mounted on Codec Fan-out optical connectors (Fig. 4).

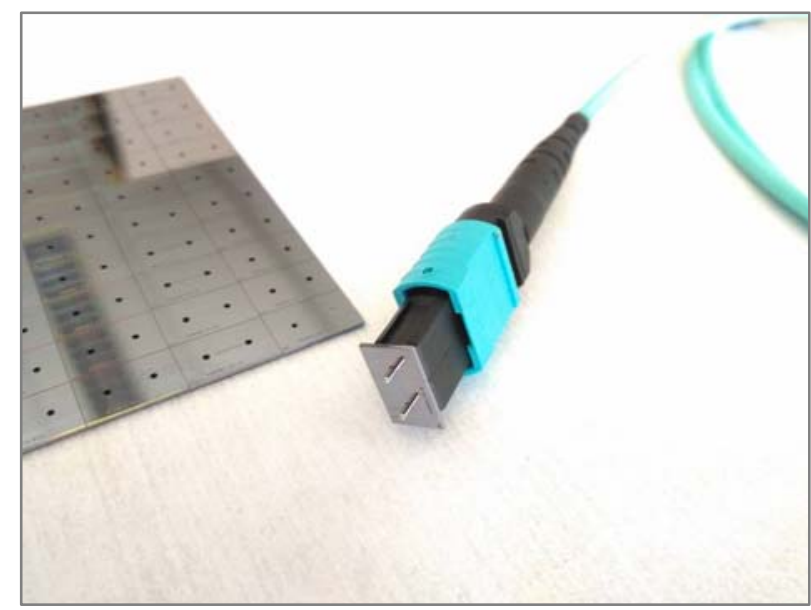

Fig. 4. Optomechanical sensor mounted on fan-out connector.

The optical tests yielded good interference patterns from the membranes by varying the wavelength of the continuous laser beam used to interrogate the cavity of the Fabry-Perot structure created in the devices by the alignment of the optical fibers in the fan-out connector and the thin sensor membranes.

In fig. 5, the result of an optical test performed on a circular membrane device mounted on the fan-out connector is reported by way of example.

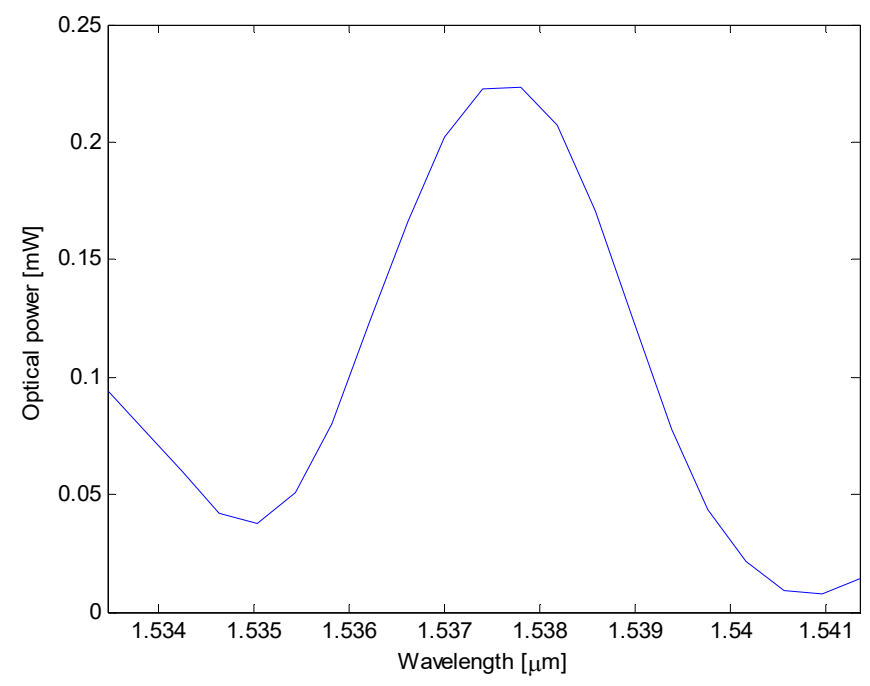

Fig. 5. Reflection spectrum from crack width measurement device mounted on optical fan-out connector.
Mechanical tests on the devices were carried out by applying a load on the membranes using a setup built in order to apply pressure to the sensor through a soft polymer layer (plasticine).

In order to apply a distributed load on the sensor, a positioning system was employed to sustain a sliding pin than could be placed on the device and apply pressure through a plasticine intermediate layer. Some screws of different size were used as additional weights in the measurements.

The results obtained are reported in Fig. 6 .

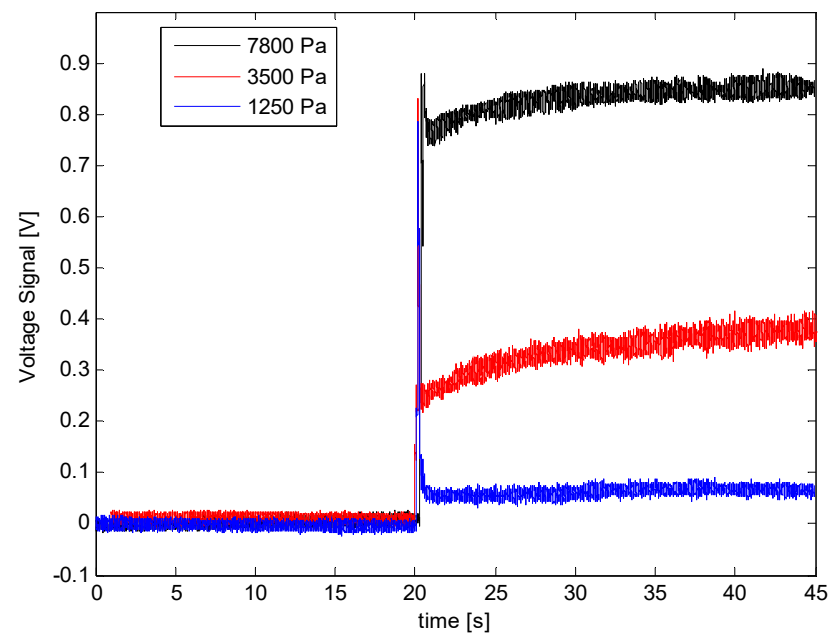

Fig. 6. Result of optomechanical test on crack width measurement device.

As may be observed, a clear optomechanical pressure signal is measured on the device upon application of the additional weights, even for the minimum load of only $1.25 \mathrm{kPa}$.

\section{ACKNOWLEDGMENT}

This work was supported by H2020-ICT-24-2015 AEROBI (AErial RObotic System for In-Depth Bridge Inspection by Contact), project. N. 687384.

\section{REFERENCES}

[1] R. Pinto, A. Medeiros, I. Padaratz, B. Andrade, "Use of Ultrasound to Estimate Depth of Surface Opening Cracks in Concrete Structures", (2010) http://www.ndt.net/?id=9954.

[2] M. Seher, C. In, J. Kim et al., "Numerical and Experimental Study of Crack Depth Measurement in Concrete Using Diffuse Ultrasound", Journal of Nondestructive Evaluation 32 (2013), pp. 81-92.

[3] L. Belsito, L. Masini, M. Sanmartin, K. Loupos, A. Roncaglia, "Ultrasonic sensor system for automatic depth measurement of surface opening cracks in concrete by means of a robotic arm", Proceedings of the International Conference on Smart Infrastructure and Construction, ICSIC 2016 (2016), pp. 239-244. 